\title{
Family of Bistable Attractors Contained in an Unstable Dissipative Switching System Associated to a SNLF
}

\author{
J. L. Echenausía-Monroy (D), J. H. García-López, R. Jaimes-Reátegui, D. López-Mancilla, \\ and G. Huerta-Cuellar iD
}

Dynamical Systems Laboratory, CULagos, University of Guadalajara, 1144 Enrique Díaz de León St., Paseos de la Montaña, Lagos de Moreno, Jalisco 47460, Mexico

Correspondence should be addressed to J. L. Echenausía-Monroy; jose.luis.echenausia@gmail.com and G. Huerta-Cuellar; g.huerta@lagos.udg.mx

Received 27 April 2018; Revised 13 July 2018; Accepted 30 July 2018; Published 15 October 2018

Academic Editor: Viet-Thanh Pham

Copyright (C) 2018 J. L. Echenausía-Monroy et al. This is an open access article distributed under the Creative Commons Attribution License, which permits unrestricted use, distribution, and reproduction in any medium, provided the original work is properly cited.

\begin{abstract}
This work presents a multiscroll generator system, which addresses the issue by the implementation of 9-level saturated nonlinear function, SNLF, being modified with a new control parameter that acts as a bifurcation parameter. By means of the modification of the newly introduced parameter, it is possible to control the number of scrolls to generate. The proposed system has richer dynamics than the original, not only presenting the generation of a global attractor; it is capable of generating monostable and bistable multiscrolls. The study of the basin of attraction for the natural attractor generation (9-scroll SNLF) shows the restrictions in the initial conditions space where the system is capable of presenting dynamical responses, limiting its possible electronic implementations.
\end{abstract}

\section{Introduction}

Over the last few years, the development and implementation of chaotic oscillators have been extensively studied, taking a special interest in the generation of systems with multiscrolls in their phase space, such as the Lorenz [1] and Chua [2] systems, which present a double-scroll attractor. There are several methods to obtain multiscroll behavior, for example, by adding breakpoints to Chua's function [3, 4], by using a system with hysteresis $[5,6]$, implementing step functions, using sine/cosine functions, or by generating piecewise linear functions [7-10]. The disadvantage of these methods is that the systems have more fixed points than scrolls generated, so increasing the number of scrolls in the phase space turns into a more complex task to address. As an alternative to this dilemma arises, the conception of a saturated nonlinear function, SNLF, which is based on the operational amplifiers performance $[11,12]$, which guarantees to find as many scrolls as segmentation points the function possesses, being a simpler way to approach the topic in the scrolls generation.

An example of this kind of systems can be described within the theory of unstable dissipative systems, UDS $[13,14]$, which characterizes the systems of three differential equations based on the location of the eigenvalues that it possesses. UDS's are classified as type I or type II, where the order of the type represents the number of eigenvalues with negative real part. In general, any three-dimensional dynamic system is considered an UDS if and only if it has a combination of eigenvalues that coincide with the definition of a hyperbolic saddle-node, and the sum of these components is negative, i.e., the dissipation condition is fulfilled [15]. Examples of these systems are found in Rössler [16], Lorenz [1], and Chua [2], among some other systems [17-19]. This kind of combination in the eigenvalues favors the appearance of multiscroll behavior, by means of the implementation of the appropriated nonlinear function. 
In recent years, the design and control of systems with multiple scrolls have been a subject of interest for the scientific community, having a great impact in their application, such as secure communication systems, neuronal modeling, and generation of pseudorandom systems [20-23]. In this work, the modification in a multiscroll dynamics by means of controlling the associated nonlinear function is presented, introducing a new control parameter. This new control parameter helps to generate a specific number of scrolls, generating regions of coexisting attractors, as well as the generation of attractors with double-wing and three equilibrium points.

This work is structured as follows: the first section contains an introduction that describes previous works and theoretical principles of the system. The second section shows the UDS definition and the description of the multiscroll generator system. In the third section, the methodology and results of the studied system are shown. The analysis of the bifurcation diagrams exhibits the coexistence of two attractors for fixed set parameters, bistability, which is illustrated by the construction of the corresponding basins of attractions. The main conclusions are shown in the last section.

\section{Theoretical Background}

2.1. Unstable Dissipative Systems Theory. In the same spirit as $[13,24,25]$, it is considered as a system of autonomous differential equations of third order,

$$
\dot{X}=A X+B f(x),
$$

where $\boldsymbol{X}=[x, y, z]^{T} \in \mathbb{R}^{3}$ is the state vector, $\boldsymbol{A}=\left[a_{i, j}\right] \in \mathbb{R}^{3 \times 3}$ is a constant matrix, $\boldsymbol{B}=\left[b_{1}, b_{2}, b_{3}\right]^{T} \in \mathbb{R}^{3}$ is a constant position vector, and $\boldsymbol{f}(\boldsymbol{x})$ is a nonlinear function. The behavior of the system is governed by the eigenvalues of the matrix $A$, which generates a great variety of characteristic values, presenting special attention to those saddle-node points that have a stable and an unstable variety. This kind of eigenvalues is responsible for both stretching and successive folding in the dynamic of the system, which favors the generation of multiscrolls $[14,26]$.

A system can be considered as an UDS type I if their equilibrium points correspond to a hyperbolic-saddle-node, i.e., one eigenvalue is negative real (dissipative component) and the other two are complex conjugated with positive real part (unstable and oscillatory component), where the sum of the components must be less than zero. By other side, an UDS type II, eq. (1), is described in the opposite way, i.e., one eigenvalue is positive real (unstable component) and the other two are complex conjugated with negative real part (dissipative and oscillatory component), and the sum of them must be less than zero [13].

2.2. Multiscroll Generator. The multiscroll generator system studied is described by a set of three coupled differential equations that makes use of the definition of a saturated nonlineal function, SNLF, as a method for the scroll generation [12, 27], eq. (2).

$$
\begin{aligned}
& \dot{x}=y, \\
& \dot{y}=z, \\
& \dot{z}=-\alpha_{1} x-\alpha_{2} y-\alpha_{3} z+\alpha_{4} f(x, q),
\end{aligned}
$$

where $x, y, z$ are the state variables, $f(x, q)$ is the SNLF, $q$ is the upper limit of scrolls to generate, and $\alpha_{1,2,3,4}$ are the system parameters that define the behavior of the dynamic. This work is focused on the region for which the system is defined as a UDS I. Within this proposition, the multiscroll appearance is possible, by generating a conservative component that causes the oscillation of the system over an equilibrium point, while the other two dissipative components favor the visit to other fixed points, resulting in the dynamics of a multiscroll system. The operation region of the system is defined by the combination of the system parameters, in this case, the following consideration is contemplated: $\alpha=\alpha_{1}=\alpha_{2}=\alpha_{3}=\alpha_{4}$.

Considering the previous condition, it is possible to examine the behavior of the equilibrium points by sweeping the control parameter and finding the operation zone where their eigenvalues are consistent with an UDS I definition. The control parameter variation is developed by means of the characteristic polynomial of the system described in eq. (2), $\lambda^{3}+\alpha\left(\lambda^{2}+\lambda+1\right)=0$, and are plotted by considering the split of the real and imaginary component of each eigenvalue, $\lambda_{j}=\sigma_{j}+i \omega_{j}, j=1,2,3$, being $\sigma_{j}$ the real part, and $\omega_{j}$ the imaginary part. Figure 1 shows the analysis of the eigenvalues over a range value defined as $\alpha=[-2,2]$, Figure 1(a) shows the operation zone delimited by $0<a<1$. Figures 1 (b) and 1 (c) confirm the conditions for the UDS I definition. $\lambda_{1}$ the real negative eigenvalue, and $\lambda_{2,3}$ the complex conjugated eigenvalues with positive real part.

It is well known $[11,12,27]$ that it is possible to define a series of saturated functions, based on the behavior of an operational amplifier (op-amp) configured as voltage comparator, in order to generate as many plateaus (commutation surfaces) as desired, and thus guarantee the generation of the same number of scrolls. In general, the mathematical construction of a SNLF is defined as

$$
f\left(x_{k, h, p, q}\right)=\sum_{m=-p}^{q} f_{m}\left(x_{k, h}\right)
$$

where $k>0$ is the slope that connects the plateaus in the saturated function, $h>2$ is the delay time of the saturated function, defined by the op-amp switching speed, $p$ and $q$ are positive integers defined as the smallest integer that result from dividing the number of scrolls by $2, m=1,2,3, \ldots, n$, where $n$ defines the number of scrolls to generate. The function $f(x)$ is defined as follows

$$
f_{m}\left(x_{k, h}\right)= \begin{cases}2 k, & f x>m h+1, \\ k(x-m h)+k, & \text { if }|x-m h| \leq 1, \\ 0, & f x<m h-1,\end{cases}
$$




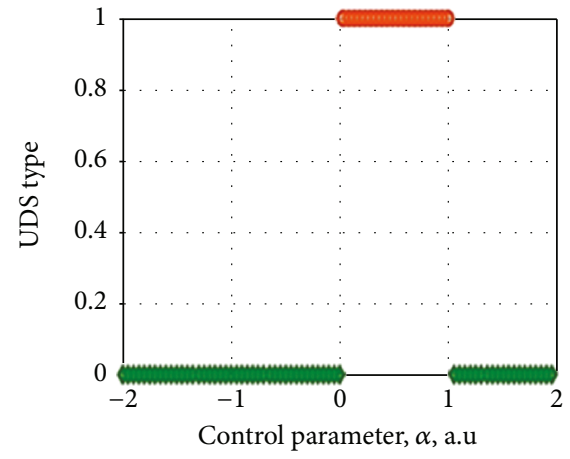

$\diamond$ NOUDS UDS I

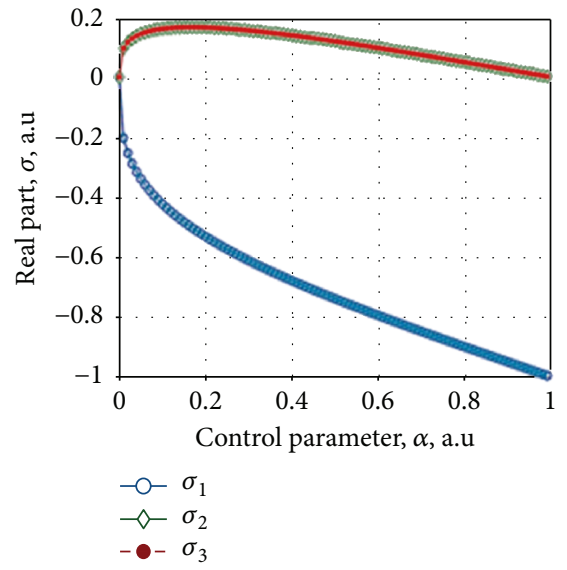

(b)

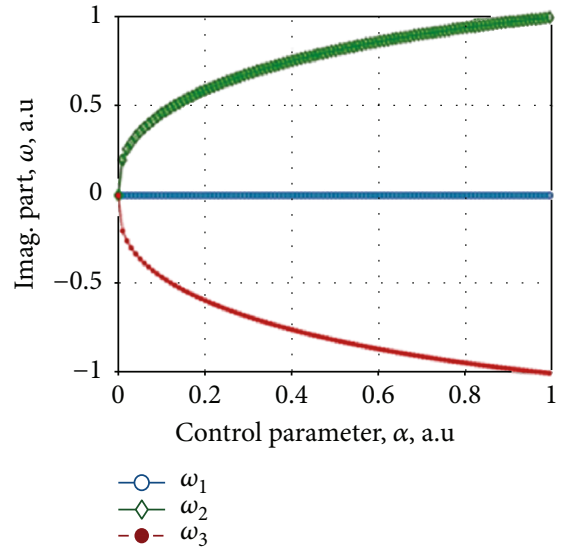

(c)

FIGURE 1: Eigenvalues analysis as a function of the control parameter $\alpha$. (a) Control parameter analysis showing the UDS I region. (b) and (c) real and imaginary parts for the UDS I, respectively.

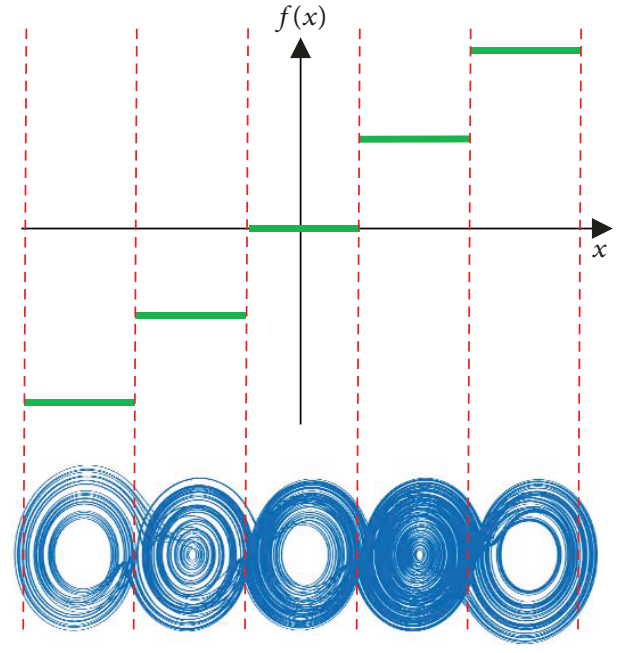

(a)

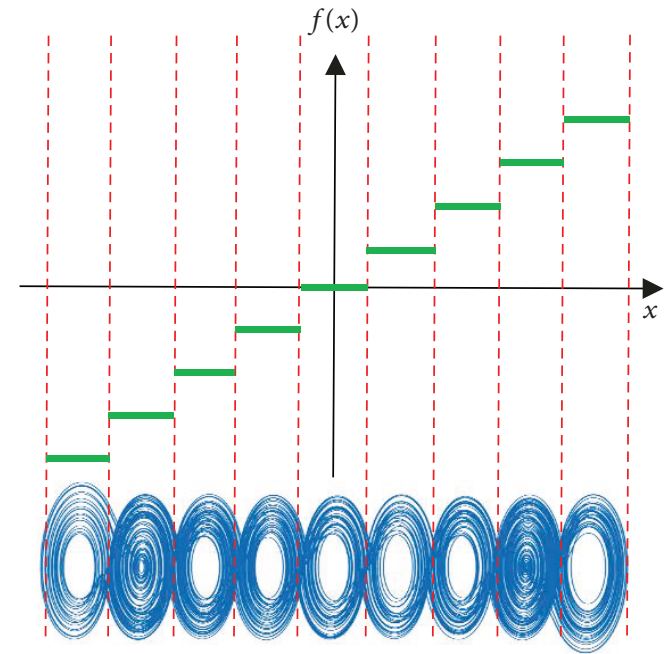

(b)

FIGURE 2: (a) Saturated nonlineal function with $n=5$ and its corresponding attractor of five multiscrolls. (b) Saturated nonlineal function with $n=9$ and its corresponding attractor of nine multiscrolls.

and

$$
f_{m-1}\left(x_{k, h}\right)= \begin{cases}0, & \text { if } x>m h \pm 1, \\ k(x \pm m h)-k, & \text { if }|x \pm m h| \leq 1 \\ -2 k, & \text { if } x<-m h-1\end{cases}
$$

Figure 2 shows the graphical representation of eq. (4) and (5) (green) and their corresponding attractor (blue). The breakpoints are plotted with a red dotted line, where the system switches between the different generated commutation surfaces. At the middle of each switching surface, the equilibrium point is located, where each scroll oscillates. Figure 2(a) SNLF generated with $n=5$, whereas Figure 2(b) shows a SNLF with $n=9$. Notice the relation between the number of switching surfaces and generated scrolls.
The SNLF contemplated for this study is constructed based on [11], eq. (4) and (5), for the generation up to nine scrolls as in Figure 2(b), but it is studied with a new bifurcation parameter $\zeta$, modifying the original system, eq. (2) $[28,29]$. This new parameter works as an individual control gain for the nonlinear function. This allows the possibility to study the bifurcation diagrams for a defined set of parameters in the model and generates more than one single attractor. The modified system is described by

$$
\begin{aligned}
& \dot{x}=y, \\
& \dot{y}=z, \\
& \dot{z}=-\alpha[x+y+z-\zeta f(x, q)],
\end{aligned}
$$

where $\alpha$ is a control parameter and $\zeta$ is the bifurcation parameter. This modification allows to analyze the 


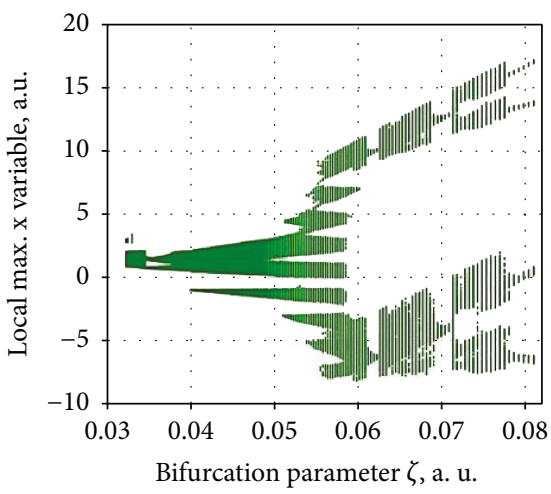

(a)

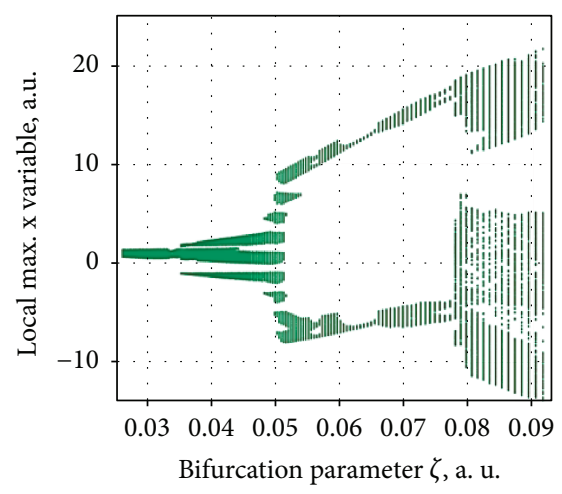

(b)

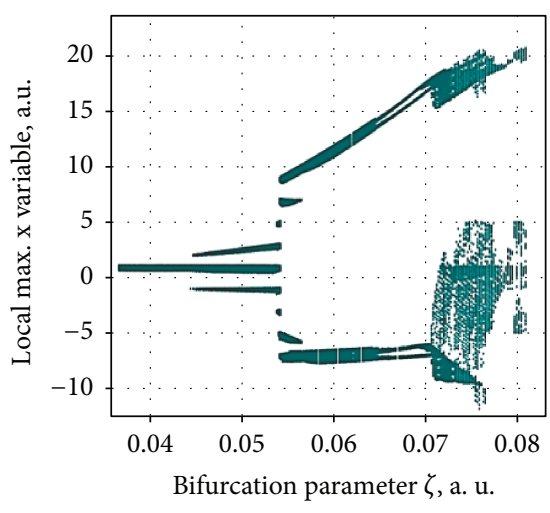

(c)

Figure 3: Bifurcation diagram for the dynamical control parameter (a) $\alpha=0.45$, (b) $\alpha=0.70$, (c) $\alpha=0.90$.

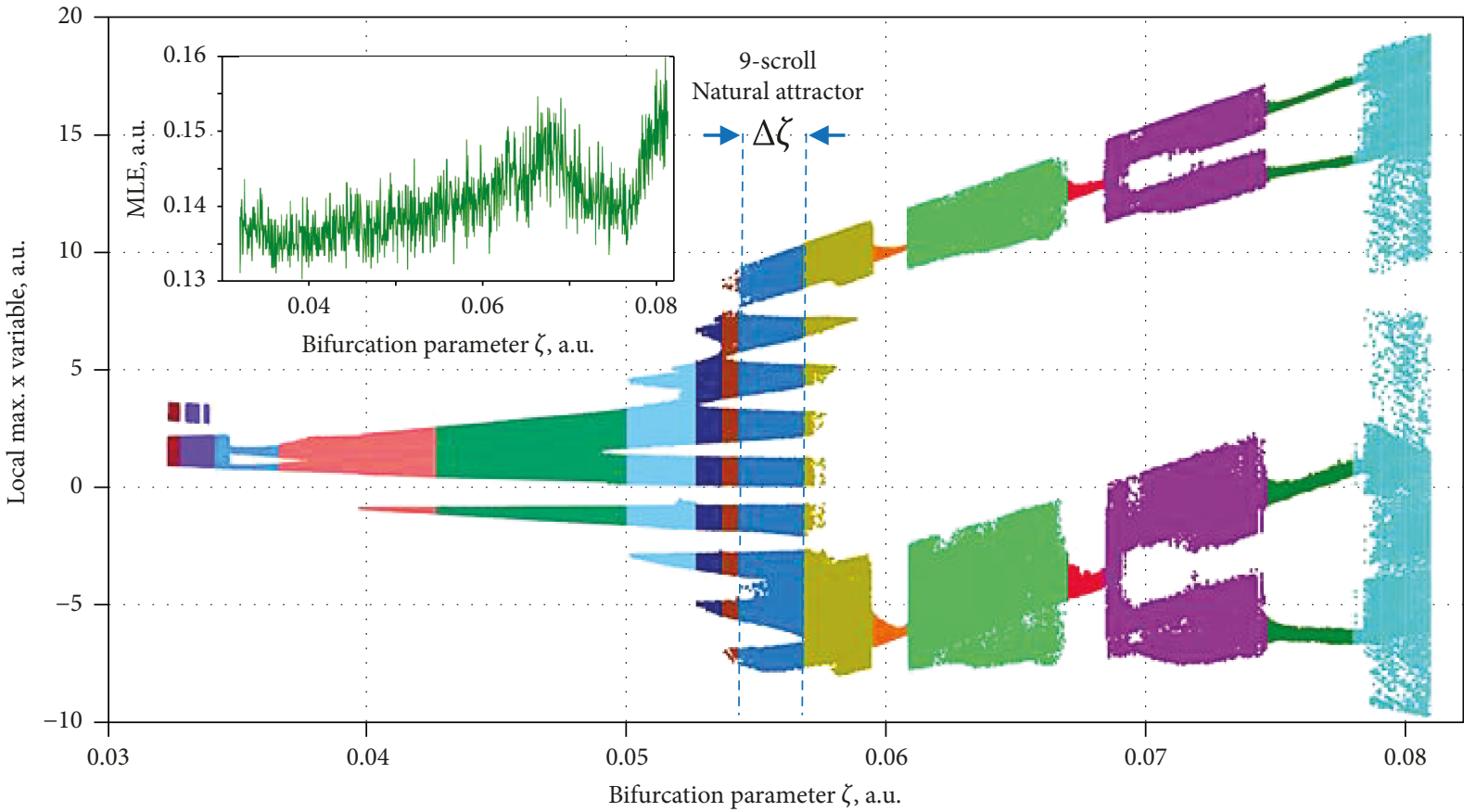

FIGURE 4: Bifurcation diagram for the dynamical control parameter $\alpha=0.45$.

behavior of the system through numerical simulation in a better way. In this case, the implementation of an $\mathrm{Rk} 4$ integrator is used.

\section{Methodology and Results}

Considering the restrictions of the system, eq. (6), given the region of operation under the conditions of an UDS I, the control parameter is analyzed in the interval $0.05 \leq \alpha \leq 0.95$ with a step size $\Delta \alpha=0.05$.

The dynamical system, eq. (6), is analyzed by a gradual change of the bifurcation parameter $\zeta$ for the different $\alpha$ values. For this purpose, the construction of bifurcation diagrams of local maximums of the state variable $x(t)$, are calculated by means of randomly changing the initial condition of the three state variables.
Analyzing the dynamical system, Figure 3, it is observed that the behavior of the different bifurcation diagrams has a dependence on the control parameter $\alpha$, but obtaining in each one of them a control in the generation of multiscrolls. However, it is more interesting to describe the dynamical evolution that the system exhibits for a value $\alpha=0.45$ in Figure 3(a). This is because the scrolls generation is produced gradually, obtaining a greater number of generated dynamics, unlike the behavior displayed in other values of the control parameter.

The 9-scroll natural attractor region $\Delta \zeta$ is shown in Figure 4. At the left top of this figure, the last largest Lyapunov exponent (MLE) is shown, corresponding to the same bifurcation diagram. Notice that it is always a positive value, in concordance with UDS I definition. This bifurcation diagram is plotted by a color combination, where each color 


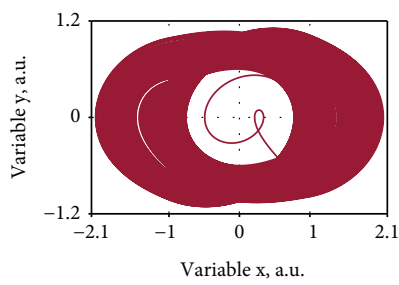

(a)

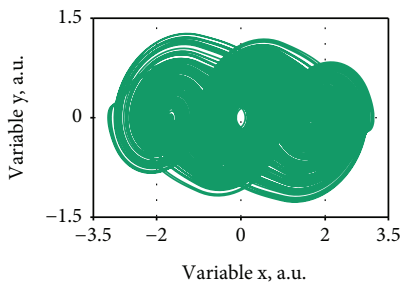

(e)

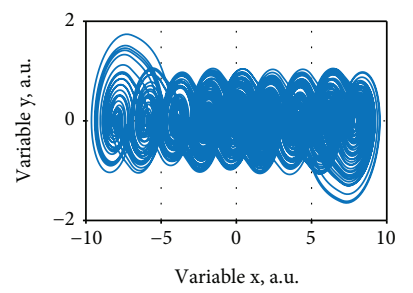

(i)

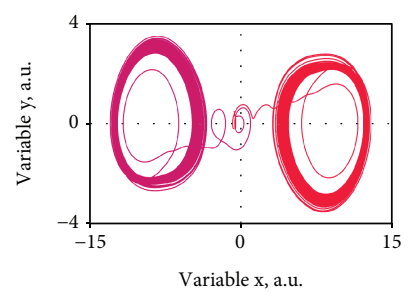

(m)

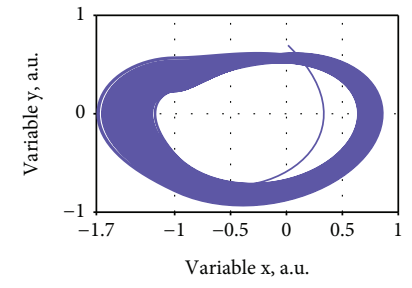

(b)

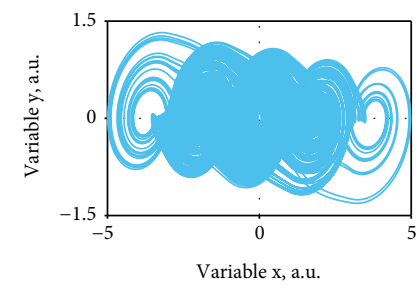

(f)

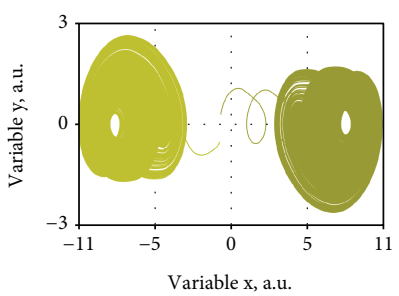

(j)

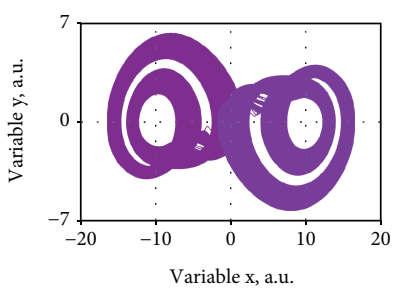

(n)

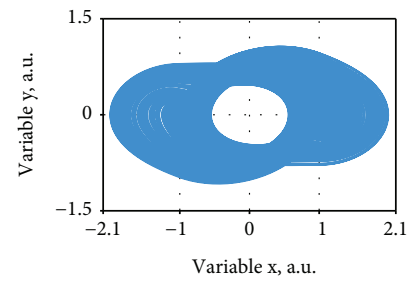

(c)

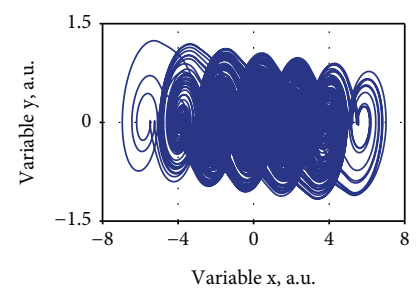

(g)

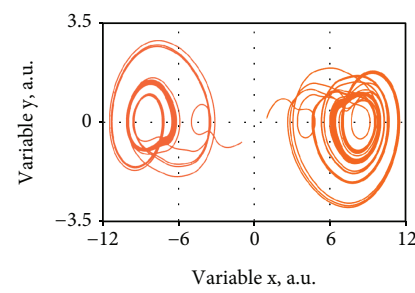

(k)

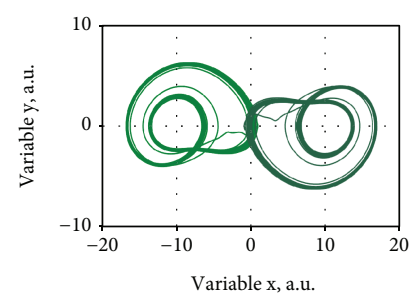

(o)

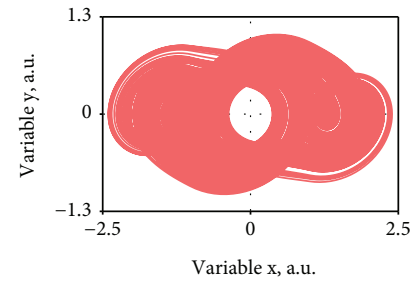

(d)

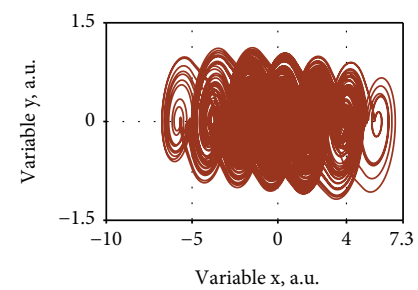

(h)

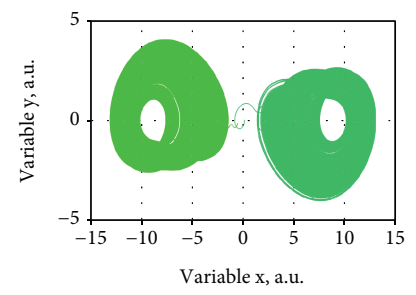

(l)

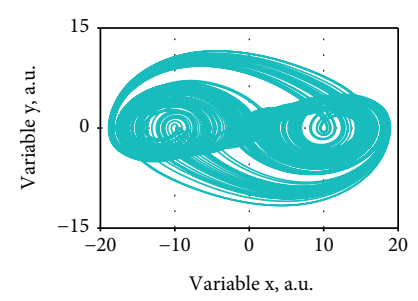

(p)

FIGURE 5: Space phase for each attractor identified into the bifurcation diagram, Figure 4, for the control parameter $\alpha=0.45$.

represents a different behavior in the system, related to the corresponding attractors displayed in Figure 5.

Table 1 describes the different behavior of the bifurcation diagram of Figure 4 as well as the corresponding attractors of Figure 5. The bistability referred in Table 1 indicates the coexistence of two identical attractors located at symmetrical equilibrium points. Notice that from Figures $5(\mathrm{j})-5(\mathrm{o})$ the bistable attractors are shown in the same phase space and plotted with different colors for easier visualization, i.e., in Figure $5(\mathrm{~m})$ the pink scroll represents the lower part of the bifurcation diagram, while the red scroll is the upper part in the same diagram for $0.0670<\zeta<0.0690$.

\section{Discussion}

With the premise that every UDS I system has large basins of attraction [30], the analysis of the dynamical system, eq. (6), is determined for each value of the dynamical parameter $\alpha$, by means of the exploration of the bifurcation parameter $\zeta$, being $\alpha=0.05$ the first explored value.

An analysis of the dynamical response of the natural system attractor (nine multiscrolls) in a bidimensional space $\alpha v s x_{c i}=y_{c i}=z_{c i}$, where $c i$ represents the initial condition, is shown in Figure 6. It can clearly be seen that meanwhile the $\alpha$ value is increased, the area of initial conditions grows (green points), while the nonexistence of the dynamical response is shown by every blue point. As a result, $\alpha=0.3$ is the minimum value in which it is possible to perform the characterization of the system, eq. (6), as defined in the previous section. This fact limits the possible electronic implementation of the model.

As in $[28,29]$, in this work, the bifurcation parameter $\zeta$ is dependent on the dynamical parameter $\alpha$, as shown in Figure 3. This effect can be visualized in Figure 7(a) that shows the ration $\Delta \zeta / \zeta(\alpha)$. As a result, the ration has a maximum point for $\alpha=0.3$. The operation region relative size, $\zeta(\alpha)$, is normalized to its $\zeta(\alpha)_{\max }$ to improve the 
TABLE 1: Behavior analysis of Figures 4 and 5.

\begin{tabular}{lccc}
\hline$\zeta$ interval & Attractor type & Bistability & Fig. \\
\hline $0.0310-0.0320$ & Single-wing deformed & No & Fo \\
$0.0320-0.0335$ & Single-wing & No & Figure 5(b) \\
$0.0335-0.0350$ & Single-wing deformed & No & Figure 5(c) \\
$0.0350-0.0420$ & Single-wing with 3 equilibrium points & No & Figure 5(d) \\
$0.0420-0.0500$ & 3-scroll & No & Figure 5(e) \\
$0.0500-0.0510$ & 5-scroll & No & Figure 5(f) \\
$0.0510-0.0520$ & 7-scroll & No & Figure 5(g) \\
$0.0520-0.0530$ & 8-scroll & No & Figure 5(i) \\
$0.0530-0.0570$ & 9-scroll (natural attractor) & Yes & Figure 5(j) \\
$0.0570-0.0590$ & Single-wing & Yes & Figure 5(k) \\
$0.0590-0.0610$ & Coherent single-wing & Yes & Figure 5(l) \\
$0.0610-0.0670$ & Single-wing & Yes & Figure 5(m) \\
$0.0670-0.0690$ & Single-wing & Yes & Figure 5(n) \\
$0.0690-0.0740$ & Coherent single-wing & Yes & Figure 5(o) \\
$0.0740-0.0780$ & Coherent single-wing & No & Figure 5(p) \\
$0.0780-0.0820$ & Double-scroll with 3 equilibrium points & &
\end{tabular}

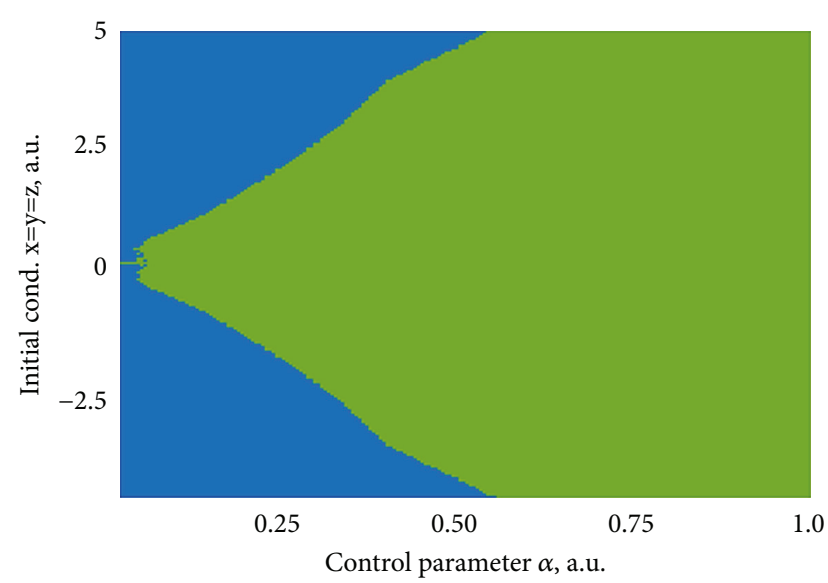

FIGURE 6: Bidimensional space $\alpha v s x_{c i}=y_{c i}=z_{c i}$ for the natural system attractor by means of the dynamical parameter modification.

visualization, $\zeta(\alpha) / \zeta(\alpha)_{\max }$, Figure $7(\mathrm{~b})$. The values $\alpha<0.3$ are omitted due to the results shown in Figure 6.

As a result of the bifurcation diagrams shown in Figures 3 and 4 and to the bistable attractors shown in Figure 5, an analysis is developed in search of bistable behavior in all the control parameters of the system for which its response is defined as an UDS I. The study in search of bistable dynamics is developed in the following way: given a control parameter $\alpha$, the bifurcation parameter $\zeta$ is fixed, and the basins of attraction of the system, eq. (6), are calculated under the modification of two of the three state variables for the space of initial conditions defined as $x, y=[-4,4], z=0$. The results shown by the basins of attraction confirm the bistable behavior for any $\alpha$ value and are validated by analyzing the attractors generated for different explored initial conditions, Figure 8, where each column corresponds to a $\alpha$ value.

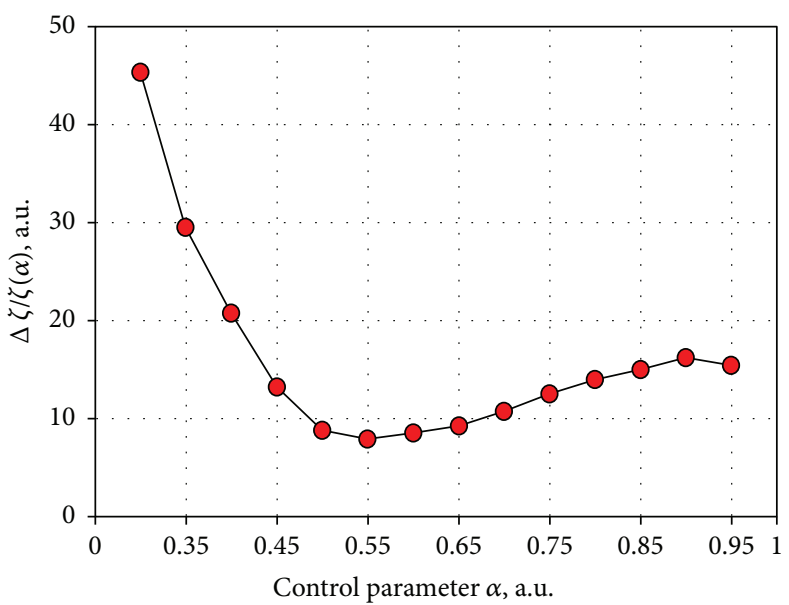

(a)

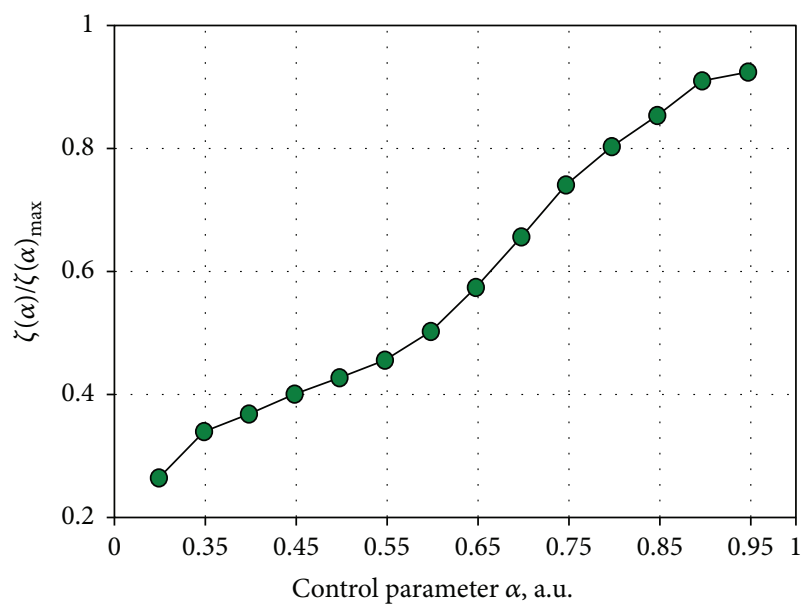

(b)

FIgURE 7: (a) Ration curve of the 9-scroll operation zone and (b) normalized operation zone for every dynamical value. 


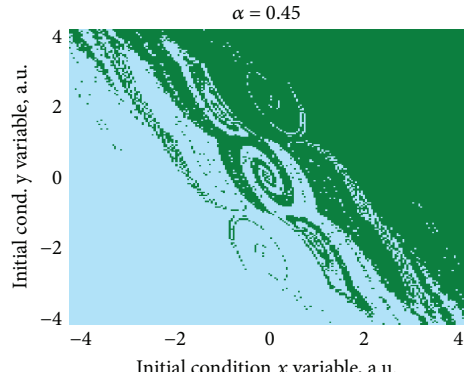

(a)

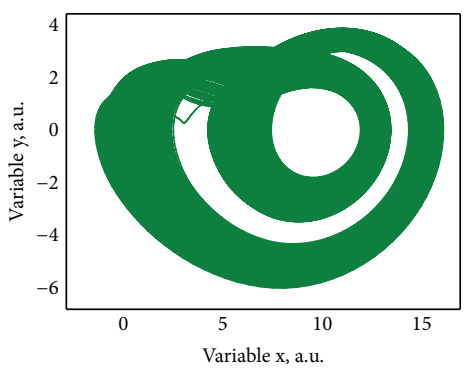

(d)

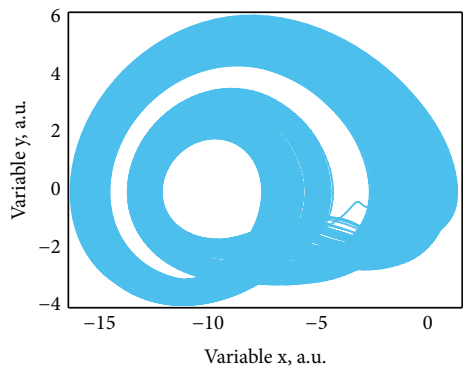

(g)

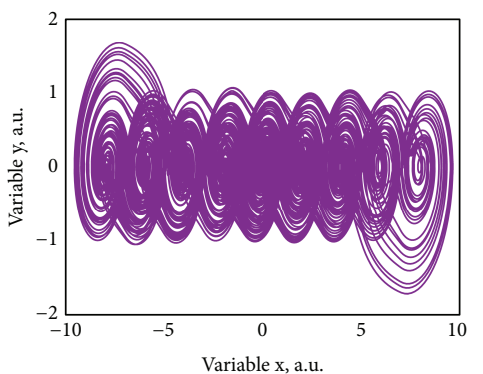

(j)

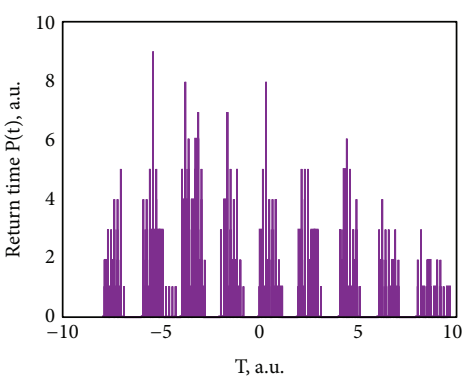

(m)

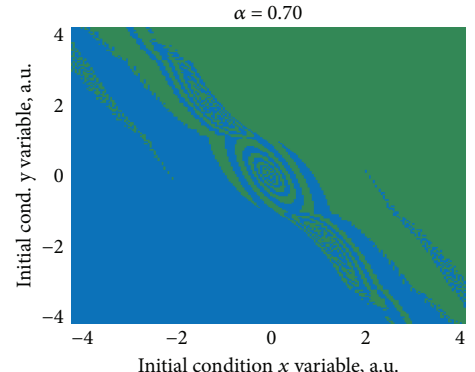

(b)

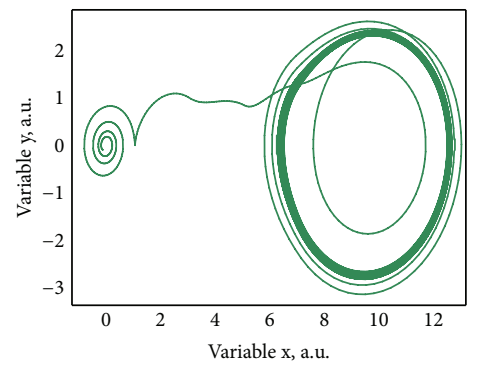

(e)

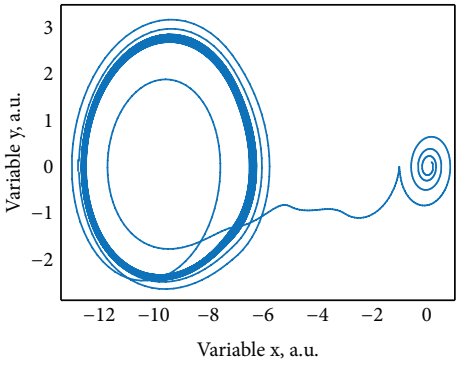

(h)

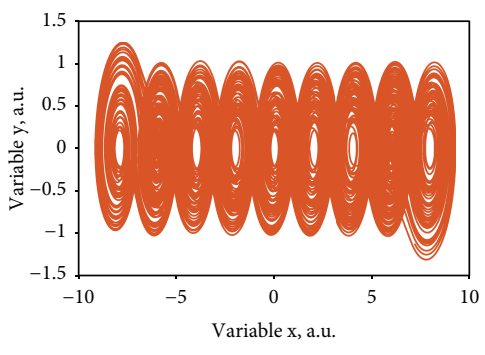

(k)

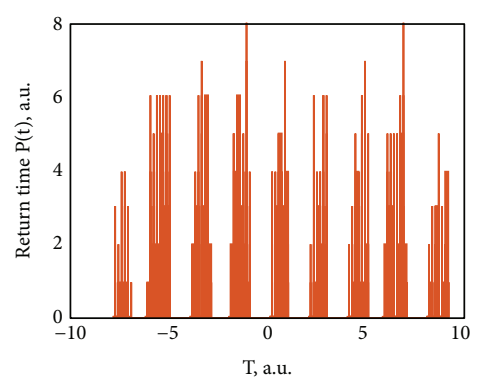

(n)

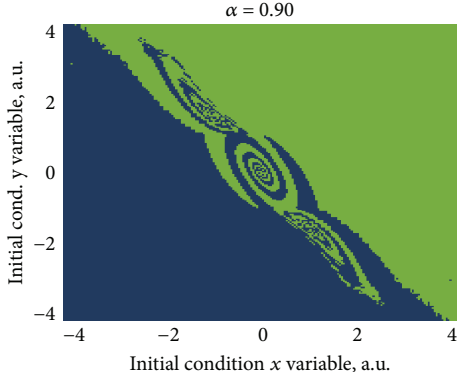

(c)

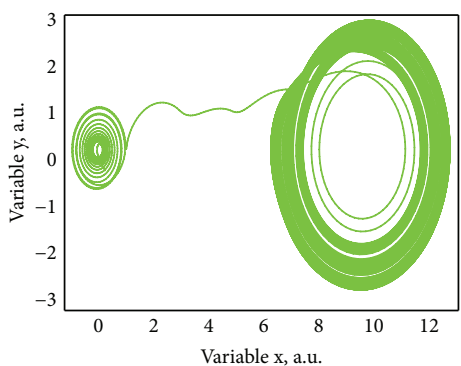

(f)

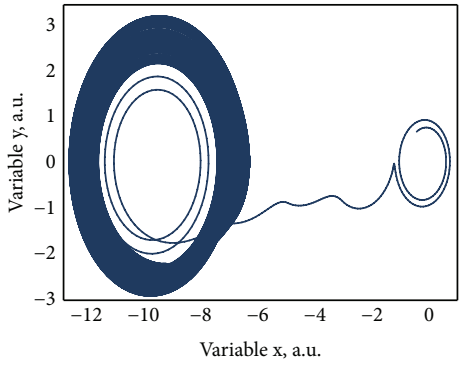

(i)

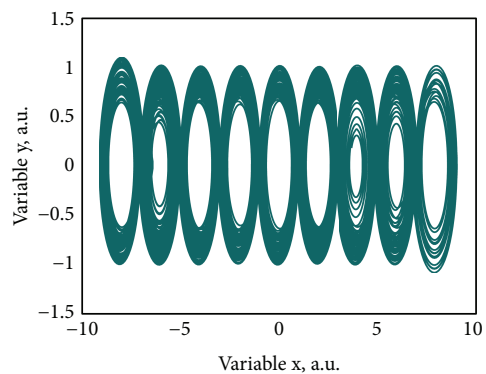

(l)

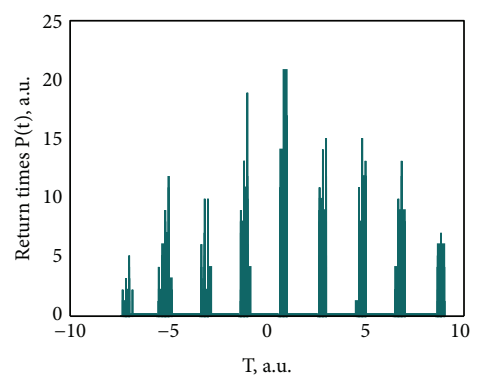

(o)

FIGURE 8: $(\mathrm{a}-\mathrm{c})$ Basins of attraction for the coexisting coherent single-wing attractor. $(\mathrm{d}-\mathrm{i})$ Coexisting attractors for each basin of attraction. (j-l) Natural system attractor. ( $\mathrm{m}-\mathrm{o}$ ) Return time distribution calculated by the Poincare section for the natural system attractor. Columns from left to right correspond to $\alpha=0.45,0.70,0.90$. 
Figures $8(a)-8(c)$ shows three different basins of attraction calculated for different $\alpha$ values under the conditions for the generation of a coexisting coherent single-wing attractor. The corresponding coexisting attractors are plotted in Figures 8(d)-8(i), where the color of the attractor is related to the color in their corresponding basin of attraction.

Analyzing the resulting basins of coexisting attractors, Figures $8(\mathrm{a})-8(\mathrm{c})$, it is evident that while the dynamical parameter of the system is increased, the basin tends to look more defined, where the state borders are better distinguished. This means that for small values in $\alpha$, the system requires a very small variation in the initial condition, or a very low noise level, to leave the actual state and converge to another coexisting equilibrium point. Meanwhile, for larger values in the dynamical parameter, the system obtains robustness by requiring significantly larger changes to leave one state and converge to another one. This phenomenon can be visualized if the return times of the attractor are calculated via the Poincaré section, from the natural attractors of each analyzed $\alpha$ value, Figures 8(j)$8(\mathrm{o})$. The distribution of these return times tends to gain some order similar to a normal distribution, Figure 8(o), becoming in softer transitions between each of the equilibrium points.

\section{Conclusions}

In this work, a multiscroll system of three-dimensional autonomous equations with a parameter $\zeta$ has been presented and studied. The parameter $\zeta$ controls the number of generated scrolls, being up to nine the maximum number of scrolls using a saturated nonlineal function. The studied system exhibits the generation of emergent behaviors from a single-wing up to a 9-scroll attractor. On the other hand, bistable states such as a single scroll, coherent single-wing, and a monostable double-scroll attractor with three equilibrium points are also obtained. The understanding of the UDS I systems has also been extended, proving that these systems have considerably large basins of attraction, but not at every value where the system fully satisfies the requirements established by the UDS theory.

The described model, in addition to involving a relatively easy implementation [27, 31], is able to control the number of scrolls to be generated, with the additional advantage of having bistable dynamics. This factor increases the potential technological applications, as in secure communication systems, neuronal systems, and control of electric motors with variable torque. The corresponding analogical implementation, in order to compare the presented results, is proposed as future work.

\section{Data Availability}

The data used to support the findings of this study are available from the corresponding author upon request.

\section{Conflicts of Interest}

The authors declare that there is no conflict of interest regarding the publication of this paper.

\section{Acknowledgments}

J. L. E. M. acknowledges CONACYT for the financial support (National Fellowship CVU-706850, No. 582124) and the University of Guadalajara, CULagos (Mexico). This work was supported by the University of Guadalajara under the project "research laboratory equipment for academic groups in optoelectronics from CULAGOS", R-0138/2016, Agreement RG /019/2016-UdeG, Mexico. The authors acknowledge J.O. Esqueda-de la Torre for his help in improving the grammar.

\section{References}

[1] E. N. Lorenz, "Deterministic nonperiodic flow," Journal of the Atmospheric Sciences, vol. 20, no. 2, pp. 130-141, 1963.

[2] L. Chua, The Genesis of Chua's Circuit, Electronics Research Laboratory, College of Engineering, University of California, Berkeley, CA, USA, 1992.

[3] M. E. Yalcin, J. A. K. Suykens, and J. Vandewalle, "Experimental confirmation of 3- and 5-scroll attractors from a generalized Chua's circuit," IEEE Transactions on Circuits and Systems I: Fundamental Theory and Applications, vol. 47, no. 3, pp. 425-429, 2000.

[4] J. A. K. Suykens, A. Huang, and L. O. Chua, "A family of $n$-scroll attractors from generalized Chua's circuit," Archiv für Elektronik und Übertragungstechnik, vol. 53, no. 3, pp. 131-137, 1997.

[5] R. W. Newcomb and N. El-Leithy, "Chaos generation using binary hysteresis," Circuits, Systems, and Signal Processing, vol. 5, no. 3, pp. 321-341, 1986.

[6] J. Lü, F. Han, X. Yu, and G. Chen, "Generating 3-D multiscroll chaotic attractors: a hysteresis series switching method," Automatica, vol. 40, no. 10, pp. 1677-1687, 2004.

[7] M. E. Yalçin, S. Özoğuz, J. A. K. Suykens, and J. Vandewalle, " $n$-scroll chaos generators: a simple circuit model," Electronics Letters, vol. 37, no. 3, pp. 147-148, 2001.

[8] W. K. S. Tang, G. Q. Zhong, G. Chen, and K. F. Man, "Generation of n-scroll attractors via sine function," IEEE Transactions on Circuits and Systems I: Fundamental Theory and Applications, vol. 48, no. 11, pp. 1369-1372, 2001.

[9] L. Chua, M. Komuro, and T. Matsumoto, "The double scroll family," IEEE Transactions on Circuits and Systems, vol. 33, no. 11, pp. 1072-1118, 1986.

[10] J. A. K. Suykens and J. Vandewalle, "Generation of n-double scrolls $(\mathrm{n}=1,2,3,4, \ldots)$," IEEE Transactions on Circuits and Systems I: Fundamental Theory and Applications, vol. 40, no. 11, pp. 861-867, 1993.

[11] J. Lü, G. Chen, X. Yu, and H. Leung, "Design and analysis of multiscroll chaotic attractors from saturated function series," IEEE Transactions on Circuits and Systems I: Regular Papers, vol. 51, no. 12, pp. 2476-2490, 2004.

[12] J. Lu, S. Yu, H. Leung, and G. Chen, "Experimental verification of multidirectional multiscroll chaotic attractors," IEEE Transactions on Circuits and Systems I: Regular Papers, vol. 53, no. 1, pp. 149-165, 2006. 
[13] E. Campos-Cantón, J. G. Barajas-Ramírez, G. Solís-Perales, and R. Femat, "Multiscroll attractors by switching systems," Chaos: An Interdisciplinary Journal of Nonlinear Science, vol. 20, no. 1, article 013116, 2010.

[14] E. Campos-Cantón, R. Femat, and G. Chen, "Attractors generated from switching unstable dissipative systems," Chaos: An Interdisciplinary Journal of Nonlinear Science, vol. 22, no. 3, article 033121, 2012.

[15] E. Ott, "Strange attractors and chaotic motions of dynamical systems," in The Theory of Chaotic Attractors, pp. 103-119, Springer, 1981.

[16] O. E. Rössler, “An equation for continuous chaos," Physics Letters A, vol. 57, no. 5, pp. 397-398, 1976.

[17] M. E. Yalçin, J. A. K. Suykens, J. Vandewalle, and S. Özoğuz, "Families of scroll grid attractors," International Journal of Bifurcation and Chaos, vol. 12, no. 1, pp. 23-41, 2002.

[18] G. Q. Zhong, K. F. Man, and G. Chen, “A systematic approach to generating n-scroll attractors," International Journal of Bifurcation and Chaos, vol. 12, no. 12, pp. 2907-2915, 2002.

[19] J. Lü, T. Zhou, G. Chen, and X. Yang, "Generating chaos with a switching piecewise-linear controller," Chaos: An Interdisciplinary Journal of Nonlinear Science, vol. 12, no. 2, pp. 344-349, 2002.

[20] J. H. Park, D. H. Ji, S. C. Won, and S. M. Lee, " $\mathscr{H}_{\infty}$ synchronization of time-delay chaotic systems," Applied Mathematics and Computation, vol. 204, no. 1, pp. 170-177, 2008.

[21] O. M. Kwon, J. H. Park, and S. M. Lee, "Secure communication based on chaotic synchronization via interval time-varying delay feedback control," Nonlinear Dynamics, vol. 63, no. 1-2, pp. 239-252, 2011.

[22] W. Yu, J. Cao, K. W. Wong, and J. Lü, "New communication schemes based on adaptive synchronization," Chaos: An Interdisciplinary Journal of Nonlinear Science, vol. 17, no. 3, article 033114, 2007.

[23] M. E. Yalcin, J. A. K. Suykens, and J. Vandewalle, "True random bit generation from a double-scroll attractor," IEEE Transactions on Circuits and Systems I: Regular Papers, vol. 51, no. 7, pp. 1395-1404, 2004.

[24] L. J. Ontañón-García, E. Jiménez-López, E. Campos-Cantón, and M. Basin, "A family of hyperchaotic multi-scroll attractors in $\mathbf{R}^{n}$," Applied Mathematics and Computation, vol. 233, pp. 522-533, 2014.

[25] A. D. Pano-Azucena, J. de Jesus Rangel-Magdaleno, E. TleloCuautle, and A. de Jesus Quintas-Valles, "Arduino-based chaotic secure communication system using multi-directional multi-scroll chaotic oscillators," Nonlinear Dynamics, vol. 87, no. 4, pp. 2203-2217, 2017.

[26] L. J. Ontañón-García, E. Jiménez-López, and E. CamposCantón, "Generation of multiscroll attractors by controlling the equilibria," IFAC Proceedings Volumes, vol. 45, no. 12, pp. 111-114, 2012.

[27] E. T. Cuautle, D. G. H. Rodríguez, J. H. Santillán, V. H. Arreola, and L. A. C. Cantera, "Simulation and experimental realization of multi-scroll chaotic oscillators," Journal of Engineering Science and Technology Review, vol. 6, no. 4, pp. 1-8, 2013.

[28] M.-S. Abdelouahab and N.-E. Hamri, "A new chaotic attractor from hybrid optical bistable system," Nonlinear Dynamics, vol. 67 , no. 1, pp. 457-463, 2012.
[29] E. Campos-Cantón, I. Campos-Cantón, J. S. González-Salas, and F. Cruz-Ordaz, "A parameterized family of single-double-triple-scroll chaotic oscillations," Revista Mexicana de Física, vol. 54, no. 6, pp. 411-415, 2008.

[30] L. J. Ontañón-García, E. Campos-Cantón, and R. Femat, "Analog electronic implementation of a class of hybrid dissipative dynamical system," International Journal of Bifurcation and Chaos, vol. 26, no. 1, article 1650018, 2016.

[31] J. L. Echenausía-Monroy, J. R. Sevilla-Escoboza, and G. Huerta-Cuéllar, "Control para la generación de multienroscados mediante la generación de interfaces virtuales," in 2 do. Congreso Iberoamericano de Instrumentación y Ciencias Aplicadas, SOMI XXXI, Guatemala, Guatemala, 2016. 


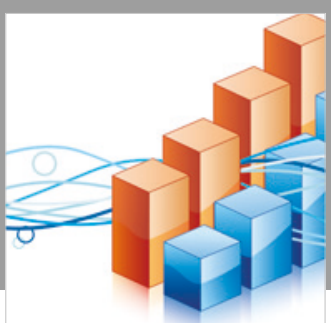

Advances in

Operations Research

\section{-n-m}
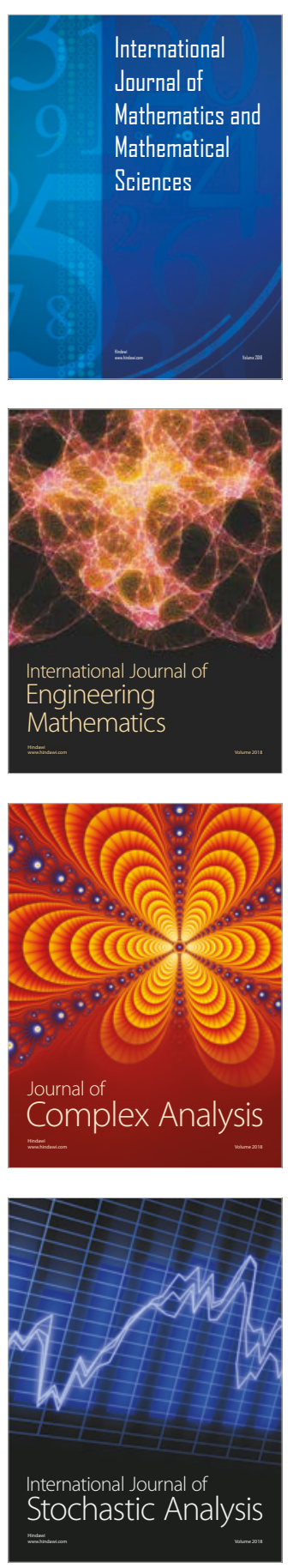
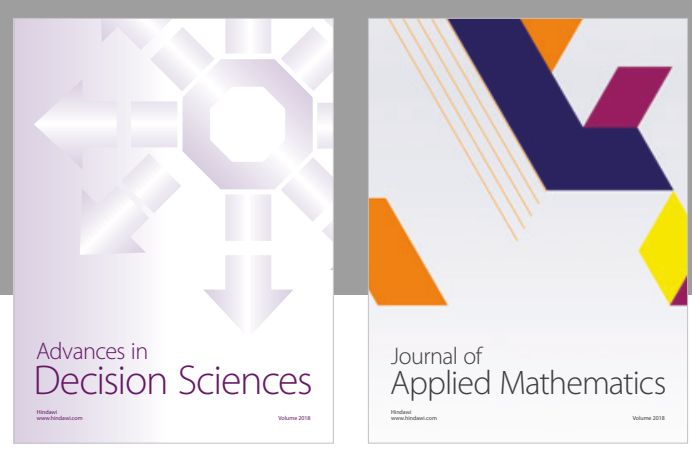

Journal of

Applied Mathematics
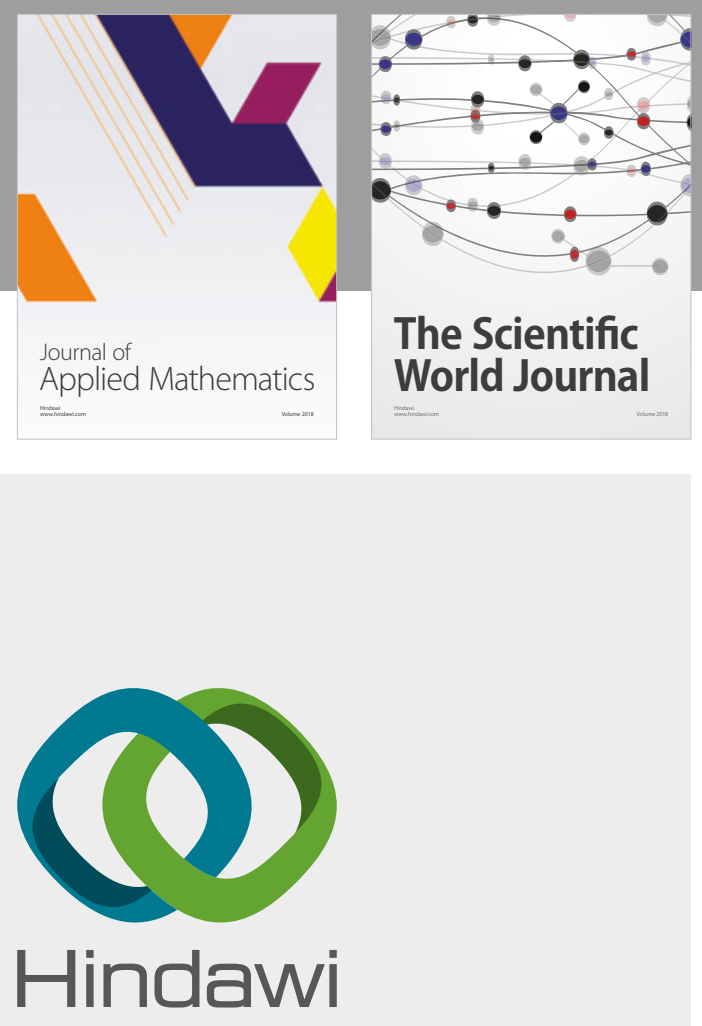

Submit your manuscripts at

www.hindawi.com

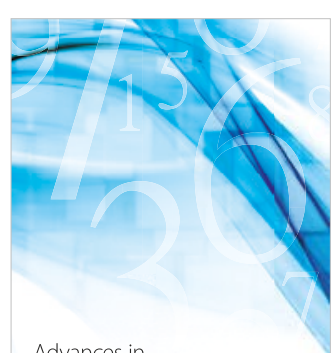

Advances in
Numerical Analysis
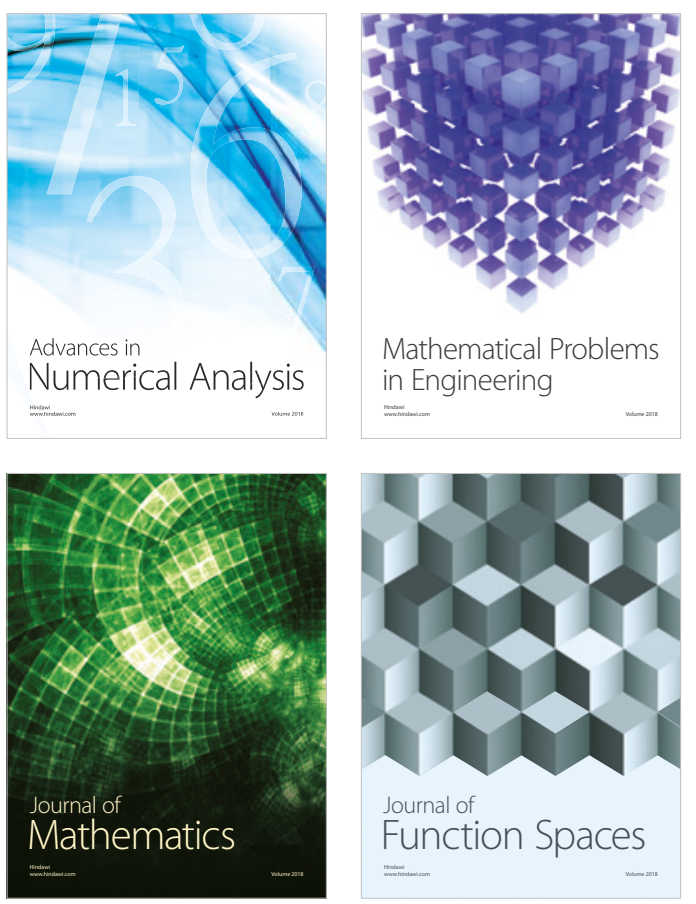

Mathematical Problems in Engineering

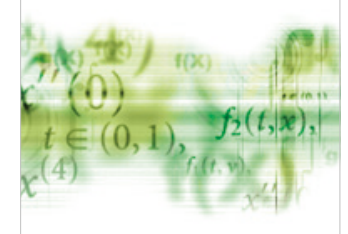

International Journal of

Differential Equations

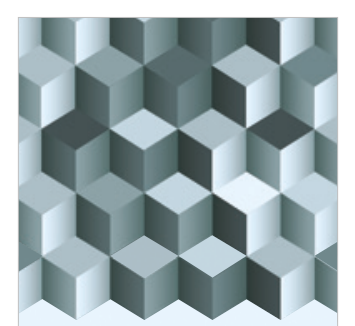

Journal of

Function Spaces
The Scientific

World Journal

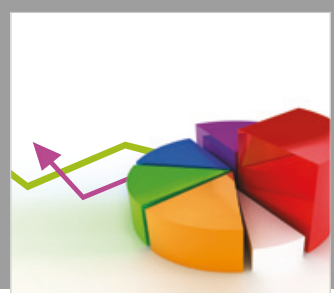

Journal of

Probability and Statistics
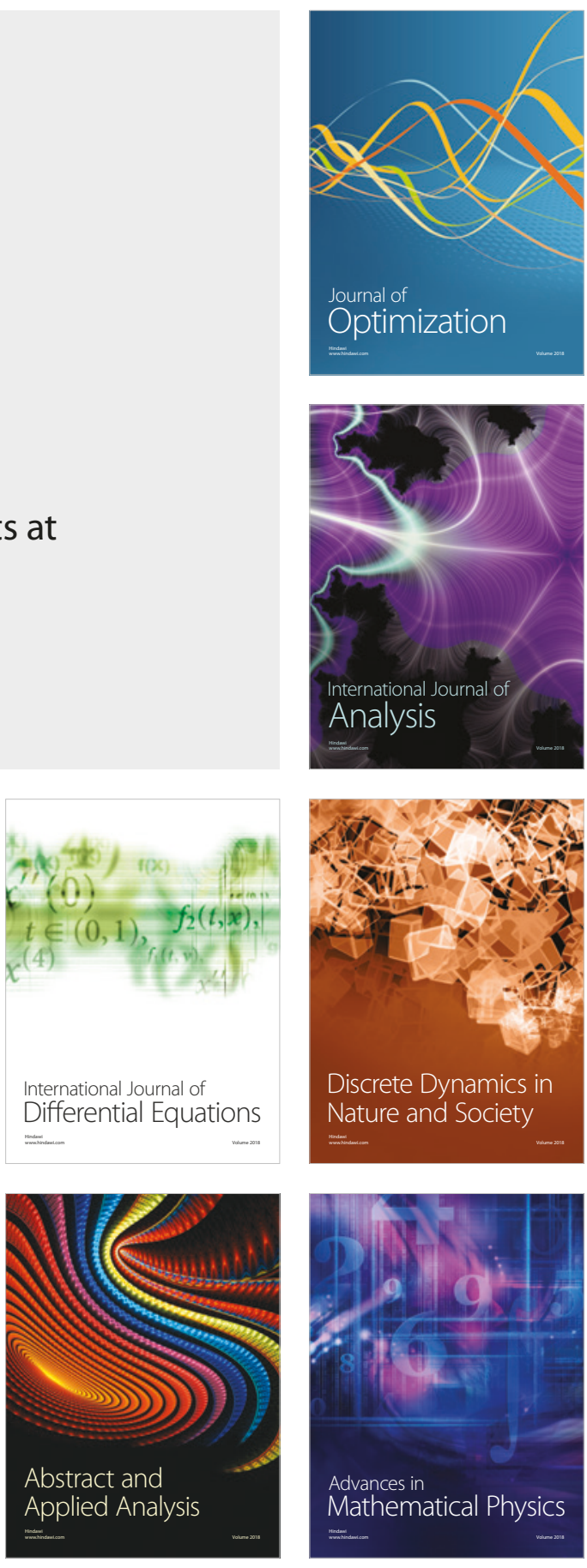